\title{
Some Results on the Intermediate Logics
}

\author{
Hiroakira ONo
}

In $[10]$, we developed the method of Kripke models and gave some applications of it to the study of the intermediate logics. We found that the use of Kripke models is very efficient, since in many cases the algebraic structure of Kripke models reflects well the properties of the logics characterized by them. In [11], we proved that a certain relation holds between the logics characterized by some Kripke models and the logics having the finite model property. As we stated in the correction at the end of $[11]$, the original proof contained an error. So, we emphasize here that the following problem remains open: Has any intermediate logic a characteristic Kripke model?

In this paper, we will proceed in the same direction as [10] and [11]. At present, we have at hand many particular intermediate logics. But we have very little knowledge about the general properties common to many logics. For instance, though many logics having the disjunction property have been known, we don't know what conditions make a logic have the disjunction property. We think that the central aim of the study of intermedate logics is to construct the theory about the general properties of them. The notion of the slice introduced by Hosoi [4] gave us the first clue to our purpose. We will introduce in $\$ 1$ other classifications of intermediate logics. In $\$ 2$, we will characterize them by Kripke models, just as the slice was characterized by the height of Kripke models in [10]. In $\S 3$, we will investigate about the disjunction property in connection with these classifications. We assume familiarity with the terminologies and the notions of $[10]$. In $[10]$, we consider a

Received October 25, 1971. 
pseudo-Boolean model not only as a lattice itself but also as the set of formulas characterized by it. To draw a clear distinction between them, we say an element of a pseudo-Boolean algebra as a value in this paper.

We wish to thank Prof. T. Hosoi for his fruitful discussions and Prof. S. Takasu for his advice.

\section{$\S 1$. Classifications of the Intermediate Logics}

In this section, we will introduce two classifications $\left\{\mathscr{T}_{n} ; 0 \leqq n \leqq \omega\right\}$ and $\left\{\mathscr{R}_{m n} ; 2 \leqq m \leqq \omega\right.$ and $\left.1 \leqq n \leqq \omega\right\}$. They have a close connection with the slice, as shown in the following.

Definition 1.1 (Ono $[10]$, Definition 4.5). $R_{2 n}(0 \leqq n \leqq \omega)$ are pseudo-Boolean models defined as

$$
\begin{aligned}
& R_{20}=S_{1}, \\
& R_{2 n}=S_{1} \uparrow S_{1}^{n} \quad \text { for } 1 \leqq n \leqq \omega .
\end{aligned}
$$

Here we restate the main theorem of [6].

Theorem 1.2 (Hosoi-Ono [6]).

1) $R_{2 n} \subsetneq R_{2 m}$ if $n>m$,

2) for each logic $L$ in $\mathscr{S}_{2}$, there is $n$ such that $L>\subset R_{2 n}$.

Now using the above theorem, we define a classification of intermediate logics similarly as the definition of the slice.

Definition 1.3. $\mathscr{T}_{n}=\left\{L ; L+P_{2} \supset \subset R_{2 n}\right\}$ for $0 \leqq n \leqq \omega$, where $P_{2}$ is the formula $\left(\left(p_{2} \supset\left(\left(\left(p_{1} \supset p_{0}\right) \supset p_{1}\right) \supset p_{1}\right)\right) \supset p_{2}\right) \supset p_{2}$.

We can verify that for any intermediate logic $L, 1)$ there exists a unique $n$ such that $L \in \mathscr{T}_{n}$, by Theorem 1.2 and 2) $L \in \mathscr{T}_{0}$ if and only if $L>C L K$. It may be expected that $\mathscr{T}_{1}=\left\{S_{n} ; 1 \leqq n \leqq \omega\right\}$. But as we show later, this is not the case. Now we show that each $\mathscr{T}_{n}$ has the greatest element and the least element. We write $(A \supset B) \wedge(B \supset A)$ as $A \equiv B$. Let $B_{n}$ be the formula 


$$
\bigwedge_{0 \leqq i \leqq n}\left(\neg p_{i} \equiv \bigvee_{\substack{j \neq i \\ 0 \leqq j \leqq n}} p_{j}\right) \supset \bigvee_{0 \leqq 1 \leqq n} p_{i} \quad \text { for } 0<n<\omega
$$

Clearly, $L J+B_{m} \ni B_{n}$ if $m \leqq n$.

Theorem 1.4. For $0<n<\omega, \mathscr{T}_{n}$ has the greatest element $R_{2 n}$ and the least element $L J+B_{n} . \mathscr{T}_{\omega}$ has the greatest element $R_{2 \omega}\left(\supset \subset L J+P_{2}\right)$ and the least element $L J$.

Proof. Clearly, $R_{2 n}(n>0)$ is the greatest element of $\mathscr{T}_{n}$ and $L J$ is the least element of $\mathscr{T}_{\omega}$. So it suffices to prove that for $0<n<\omega$, $L J+B_{n}$ is the least element of $\mathscr{T}_{n}$. First, we note that $R_{2(n+1)}=S_{1} \uparrow S_{1}^{n+1}$ and that each value of $S_{1}^{n+1}$ is of the form $\left(x_{1}, \ldots, x_{n+1}\right)$, where $x_{i}$ $\in\{0,1\}$ for any $i \leqq n+1$. Define a function $f$ from $S_{1}^{n+1}$ to the power set of $\{1,2, \ldots, n+1\}$ by

$$
f\left(\left(x_{1}, \ldots, x_{n+1}\right)\right)=\left\{k ; x_{k}=1\right\} .
$$

Now we define a formula $E(a)$ for any value $a$ in $R_{2(n+1)}$ by

$$
E(a)=\left\{\begin{array}{ll}
(p \supset p) & \text { if } a=1, \\
\bigvee_{i \in f(a)} p_{i} & \text { if } a \in S_{1}^{n+1} \\
\neg(p \supset p) & \text { if } a \neq 0,
\end{array} \text { and } a=0,\right.
$$

where 1 (or 0 ) denotes the greatest (or the least) value of $R_{2(n+1)}$. Let $C_{n}$ be the formula $\bigwedge_{0 \leqq i \leqq n}\left(\neg p_{i} \equiv \bigvee_{\substack{j \neq i \\ 0 \leqq j \leqq n}} p_{j}\right)$. Then $B_{n}$ is $C_{n} \supset E(2)$, where 2 denotes the greatest value of the subset $S_{1}^{n+1}$ of $R_{2(n+1)}$. We define an expression (by values of $R_{2(n+1)}$ ) and a function $F$ from the set of all expressions to the set of formulas as follows: For any value $a$ in $R_{2(n+1)}$, $a$ is an expression and $F(a)=E(a)$. If both $e_{1}$ and $e_{2}$ are expression, then $e_{1} \cup e_{2}, e_{1} \bigcap e_{2}, e_{1} \supset e_{2}$ and $e_{1}^{\prime}$ are expressions (where $\cup, \bigcap, \supset$ and ' denote lattice operations), and $F\left(e_{1} \bigcup e_{2}\right)=F\left(e_{1}\right) \bigvee F\left(e_{2}\right), F\left(e_{1} \cap e_{2}\right)$ $=F\left(e_{1}\right) \wedge F\left(e_{2}\right), F\left(e_{1} \supset e_{2}\right)=F\left(e_{1}\right) \supset F\left(e_{2}\right)$ and $F\left(e_{1}^{\prime}\right)=\neg F\left(e_{1}\right)$. Now we have the following lemma.

Lemma 1.5. For any expression $e, C_{n} \supset(F(e) \equiv E(e))$ is provable in 
the intuitionistic logic LJ. (Note that each expression denotes a value of $R_{2(n+1) \text {.) }}$

Now, we prove Theorem 1.4 by using the above lemma. First we remark that $L J+B_{n} \in \mathscr{T}_{n}$, since $B_{n} \in R_{2 n}$ and $B_{n} \notin R_{2(n+1)}$. Let $L$ be any logic in $\mathscr{T}_{n}$ such that $B_{n} \notin L$. Let $A$ be any formula in $L K$ but not in $R_{2(n+1)}$. Then it can be easily verified that there exists an assignment $g$ of $R_{2(n+1)}$ such that $g(A)=2$. Let $p_{1}, \ldots, p_{m}$ be all the propositional variables appearing in $A$ and let $g\left(p_{i}\right)=a_{i}$ for each $i \leqq m$. We write $e$ for the expression obtained from $A$ by replacing each $p_{i}$ by $a_{i}$ for any $i \leqq m$ and each logical connective by the corresponding lattice operation. Clearly, $e=g(A)=2$. So, by Lemma $\left.1.5 C_{n}\right)(F(e) \equiv E(2))$ is in $L J$. Hence $F(e) \supset\left(C_{n} \supset E(2)\right)$ or equivalently $F(e) \supset B_{n}$ is also in $L J$. Since $F(e)$ is a substitution instance of $A$, so if $A \in L$ then $F(e) \in L$ and hence $B_{n} \in L$. This contradicts the hypothesis. Thus $A \notin L$. Since $A$ is taken arbitrarily, it follows that $L \subset R_{2(n+1)}$. But this implies $L+P_{2} \subset R_{2(n+1)}$, which contradicts that $L \in \mathscr{T}_{n}$. Thus $B_{n}$ must be in $L$. Hence $L J+B_{n}$ $\subset L$ for any $L \in \mathscr{T}_{n}$. Now, our proof of Theorem 1.4 is completed.

Definition 1.6. $\mathscr{R}_{m n}=\mathscr{S}_{m} \cap \mathscr{T}_{n}$ for $2 \leqq m \leqq \omega$ and $1 \leqq n \leqq \omega$.

Corollary 1.7. For $2 \leqq m \leqq \omega$ and $1 \leqq n \leqq \omega, \mathscr{R}_{m n}$ has the greatest element $S_{m} \bigcap R_{2 n}$ and the least element $L J+P_{m}+B_{n}$, where both $P_{\omega}$ and $B_{\omega}$ denote the formula $p \supset p$ for the sake of brevity.

Now, we give some examples.

1) Any logic of the form $\Delta(L)$ (see Hosoi [5]) is in $\mathscr{T}_{\omega}$, since $\Delta(L) \subset \Delta(L K)>C L J+P_{2}$.

2) Let $L$ be the logic introduced by Jankov [7]. Then $L$ is in $\mathscr{R}_{\omega \omega}$. According to his result, there exist uncountably many logics $L^{\prime}$ such that $L J \subset L^{\prime} \subset L$. Thus $\mathscr{R}_{\omega \omega}$ has uncountably many elements.

3) A pseudo-Boolean model $R_{m n}$ defined in $[10]$ is in $\mathscr{R}_{m n}$ for $2 \leqq m<\omega$ and $1 \leqq n \leqq \omega$, and $D_{n-1}\left(\supset<\bigcap_{m<\omega} R_{m n}\right)$ is in $\mathscr{R}_{\omega n}$. As we have shown in Theorem 4.10 of $[10], \bigcap_{n<\omega} R_{m n}$ is the least element of $\mathscr{S}_{m}$. So, we conjectured that $\bigcap_{m<\omega} R_{m n}$ might be the least element of $\mathscr{T}_{n}$. But 


$$
\bigcap_{m<\omega} R_{m 1} \supset<S_{\omega} \supsetneq L J+\neg p \vee \neg \neg p>\subset L J+B_{1} .
$$

In general, we can prove that $\bigcap_{m<\omega} R_{m n}>\subset D_{n-1} \supseteq L J+B_{n}$ for $1 \leqq n<\omega$. Define a pseudo-Boolean model $U_{n}$ by

$$
U_{n}=S_{1} \uparrow S_{1}^{\omega} \uparrow S_{1}^{n} \quad \text { for } 1 \leqq n<\omega .
$$

Then it is easy to see that $B_{n} \in U_{n}$ but $D_{n-1} \not \subset U_{n}$ (see $\S 2$ ). Thus $D_{n-1} \not \subset L J+B_{n}$.

Definition 1.8. An intermediate logic $L$ is said to be a predecessor of a logic $L^{\prime}$ if $L \subset L^{\prime}$. A predecessor $L$ of $L^{\prime}$ is said to be immediate if $L \supsetneq L^{\prime}$ and there are no logics between $L$ and $L^{\prime}$.

We write $L<L^{\prime}$ if $L$ is an immediate predecessor of $L^{\prime}$. By the following theorem, we can see that the introduction of the classification $R_{m n}$ is suitable.

Theorem 1.9. Let $L \in \mathscr{R}_{m n}(m, n<\omega)$. Then the immediate predecessors of $L$ not in $\mathscr{R}_{m n}$ are $L \bigcap R_{(m+1) 1}\left(\in \mathscr{R}_{(m+1) n}\right)$ and $L \bigcap R_{2(n+1)}$ $\left(\in \mathscr{R}_{m(n+1)}\right)$.

Proof. We first show that if $L^{\prime} \subset L$ and $L^{\prime} \notin \mathscr{S}_{m}$ then $L^{\prime} \subset L \bigcap R_{(m+1) 1}$. Suppose that $L^{\prime} \in \mathscr{S}_{m^{\prime}}$ for $m^{\prime}>m$. Then $L^{\prime} \supset \subset L^{\prime} \bigcap R_{m^{\prime} 1}$ $C L^{\prime} \bigcap R_{(m+1) 1} \subset L \bigcap R_{(m+1) 1}$. We next show that $L \bigcap R_{(m+1) 1}$ is really an immediate predecessor of $L$. Suppose that $L \bigcap R_{(m+1) 1} \subset L^{\prime \prime} \subset L$. If $L^{\prime \prime} \notin \mathscr{S}_{m}, L^{\prime \prime} \supset \subset L \bigcap R_{(m+1) 1}$ as we have proved in the above. If $L^{\prime \prime} \in \mathscr{S}_{m}$ then

$$
L^{\prime \prime} \supset L^{\prime \prime} \bigcup\left(L J+P_{m}\right) \supset\left(L \bigcap R_{(m+1) 1}\right) \bigcup\left(L J+P_{m}\right) \supset \subset L \bigcap R_{m 1} \supset \subset L .
$$

So, $L^{\prime \prime} \supset \subset L$. Thus $L \bigcap R_{(m+1) 1}<L$. We can prove similarly that $L \bigcap R_{2(n+1)}$ is the only immediate predecessor of $L$ not in $\mathscr{T}_{n}$.

We remark here about Hosoi's theorem on the immediate predecessors of $S_{n}\left(\supset \subset R_{n 1}\right)$ (unpublished). He showed that for any $n<\omega$, if $L^{\prime}<S_{n}$ then either $L^{\prime} \supset \subset S_{n+1}>\subset S_{n} \cap R_{(n+1) 1} \quad$ or $\quad L^{\prime} \supset \subset S_{n} \cap R_{22}$ or $L^{\prime} \supset S_{n} \bigcap S_{1} \uparrow S_{1}^{2} \uparrow S_{1}\left(\in \mathscr{R}_{n 1}\right)$. 


\section{§ 2. Characterization by Kripke Models}

In this section, we will give a characterization $\mathscr{T}_{n}$ 's by using Kripke models. As a corollary, we can show that the logic $L J+B_{n}$ has the finite model property for each $n$.

We first cite a theorem in Ono [10], which gives a characterization of the slice.

Definition 2.1. Let $M$ be any Kripke model. Define $h(M)$ by the maximal length of strictly ascending sequences in $M$, if there is. Otherwise, let $h(M)=\omega$.

Theorem 2.2 (Ono $[10]$ ). Let $M$ be any Kripke model. Then for any $n \leqq \omega, h(M)=n$ if and only if $L(M) \in \mathscr{S}_{n}$.

Now, we investigate a characterization of $\mathscr{T}_{n}$ 's. It suffices to consider the case $n \geqq 1$, since $\mathscr{T}_{0}=\mathscr{S}_{1}$. However, we have no way to take the logics in $\mathscr{S}_{\omega}$ into consideration (cf. Theorem 2.4).

Let $M$ be a Kripke model. An element $a$ in $M$ is said to be maximal in $M$ if $a \leqq b$ implies $b=a$ for any $b \in M$. Define $a<b$ if $a<b$ and if $a<c \leqq b$ implies $c=b$ for any $c \in M$. We set $I(a)=\{b ; a<b\}$. Let $b$, $c \in I(a)$. We define

$b \sim c$ if there is $d \in M$ such that $b<d$ and $c<d$, and define

$b \cong c$ if there are $b_{1}, \ldots, b_{m}(m \geqq 1)$ in $I(a)$ such that $b_{1}=b, b_{m}=c$ and $b_{i} \sim b_{i+1}$ for $1 \leqq i<m$.

Clearly, $\cong$ is an equivalence relation over $I(a)$. We remark here that if $h(M)$ is finite then $I(a)$ is nonempty for any non-maximal element $a$ in $M$.

Next we define a function $w^{*}$ only for Kripke models whose height are finite and are greater than 1 , since we don't consider the logics in $\mathscr{S}_{1}$.

Definition 2.3. Let $M$ be a Kripke model such that $1<h(M)<\omega$. First we define a mapping $c$ from non-maximal elements of $M$ to 
$\{n ; 1 \leqq n \leqq \omega\}$ by

$$
c(a)=\min \{\omega, \text { the cardinality of the quotient set } I(a) / \cong\} .
$$

We set $w^{*}(M)=\sup \{c(a) ; a$ is not maximal in $M\}$. By the assumption on the height of $M, w^{*}(M)$ can be always defined.

Theorem 2.4. Let $M$ be a Kripke model such that $1<h(M)<\omega$. Then for any $n \leqq \omega, w^{*}(M)=n$ if and only if $L(M) \in \mathscr{T}_{n}$.

Proof. In the rest of this section, we write $R_{2 k}$ for the Kripke model $S_{1} \uparrow S_{1}^{k}$ (not the pseudo-Boolean model) (cf. Ono [10]). Let $w^{*}(M)=n$. We will show that $L(M) \subset L\left(R_{2 n}\right)$ and that $L J+B_{n} \subset L(M)$ only when $n<\omega$. We first prove that $L(M) \subset L\left(R_{2 c(a)}\right)$ for any nonmaximal element $a \in M$. Let $M_{a}$ be the Kripke model $\{b \in M ; a \leqq b\}$. Then it is obvious that $L(M) \subset L\left(M_{a}\right)$. Let $\sigma$ be the cardinality of $I(a) / \cong$. Define a Kripke model $N$ by $\{a\} \bigcup\left\{b_{\rho} ; \rho<\sigma\right\}$ (with the ordering $\leqq$ of $M$ ), where each $b_{\rho}$ is the representative of each equivalence class of $I(a)$. Now, we prove that $L(N)>C L\left(R_{2 c(a)}\right)$. When $\sigma \leqq \omega$, it is trivial since $\sigma=c(a)$. Let $\sigma>\omega$. One can verify that $L(N) \subset L\left(R_{2 c(a)}\right)$ and $L(N) \in \mathscr{S}_{2}$. But $L\left(R_{2 c(a)}\right)\left(\supset \subset L\left(R_{2 \omega}\right)\right)$ is the least element in $\mathscr{S}_{2}$, so $L(N) \supset L\left(R_{2 c(a)}\right)$. It remains to verify that $L\left(M_{a}\right) \subset L(N)$. Define a function $f$ from $M_{a}$ to $N$ by

$$
f(d)= \begin{cases}a & \text { if } d=a, \\ b_{\rho} & \text { if there is } b \text { such that } b \cong b_{\rho} \text { and } b \leqq d .\end{cases}
$$

Then we can show that $f$ is well-defined and that $f$ is an embedding of $M_{a}$ into $N$. Thus $L\left(M_{a}\right) \subset L(N)$ by Theorem 2.11 of [10]. Hence $L(M) \subset L\left(R_{2 c(a)}\right)$. Thus $L(M) \subset \bigcap L\left(R_{2 c(a)}\right)>C L\left(R_{2 n}\right)$, where $a$ runs over all the non-maximal elements in $M$. Next, suppose that $B_{n}$ is not valid in $M$. Then $W\left(B_{n}, b\right)=f$ for some $M$-valuation $W$ and $b \in M$. Let $a$ be a maximal element in the set $\left\{b ; W\left(B_{n}, b\right)=f\right\}$. Since $h(M)$ is finite, there exists such an $a$. We remark that $a$ is not maximal in $M$. Now, for any $0 \leqq i \leqq n, W\left(\neg p_{i} \equiv \bigvee_{j \neq i} p_{j}, a\right)=t$ and $W\left(p_{i}, a\right)=f$. So it is easy to verify that for any $i \leqq n$ there is $b_{i} \in M$ such that 
1) $W\left(p_{i}, b_{i}\right)=t$ and $a<b_{i}$ and 2) $b_{i} \neq b_{j}$ if $i \neq j$. Moreover, we can show that $a<b_{i}$ for each $i$, by the maximality of $a$. We can prove by using again the maximality of $a$ that if $b \in I(a)$ and $b \cong b_{i}$ then

$$
W\left(p_{j}, b\right)= \begin{cases}t & \text { if } j=i \\ f & \text { otherwise }\end{cases}
$$

So we can infer that if $i \neq j$ then $b_{i} \not b_{j}$. Thus $c(a) \geq n+1$. But this leads to a contradiction. Hence $B_{n} \in L(M)$. Thus we have that if $w^{*}(M)=n$ then $L(M) \in \mathscr{T}_{n}$. Conversely, suppose that $L(M) \in \mathscr{T}_{n}$ and $w^{*}(M)=m$. Then $L(M) \in \mathscr{T}_{m}$. So, $m=n$.

We will next show that the logic $L J+B_{n}$ has the finite model property for each $1 \leqq n<\omega$. As a corollary, we will show in the next section that $L J+B_{n}$ has the disjunction property for each $2 \leqq n<\omega$.

Lemma 2.5. Let $L$ be any intermediate logic such that a formula $A_{i}$ is not in $L$ for any $i \in I$. Then there is a Kripke model $M$ and an $M$-valuation $W$ such that for any $A \in L, A$ is valid in $(M, W)$ but no $A_{i}$ 's are valid in it.

Proof. Let $P$ be the Lindenbaum algebra of $L$. We write $\backslash B \backslash$ for the element of $P$ which represents the equivalence class of the formula $B$. Define an assignment $f$ of $P$ by $f(p)=\lfloor p\rfloor$ for any propositional variable $p$. Then we can verify that for any formula $B f(B)=1$ if and only if $B \in L$. Now, our lemma is immediate from Theorem 1.2 in Ono $[10]$.

Theorem 2.6. Let $\left\{M_{k} ; k<\omega\right\}$ be an enumeration of all the finite Kripke models $M$ such that $w^{*}(M) \leqq n$. Then

$$
L J+B_{n} \supset \bigcap_{k<\omega} L\left(M_{k}\right)
$$

Thus $L J+B_{n}$ has the finite model property for each $n<\omega$.

Proof. We use the method due to Segerberg [12] and Gabbay [2]. 
Let $A$ be any formula not in $L J+B_{n}$. Then by Lemma 2.5, there is a Kripke model $M$ and an $M$-valuation $W$ such that each substitution instance of $B_{k}(k \geqq n)$ is valid in $(M, W)$ but $A$ is not valid in it. Let $K_{0}$ be the set of all subformulas of $A$, and $K$ be the closure of $K_{0}$ under the connectives of negation, conjunction and implication. Define $\equiv$ by the condition that for each $a, b \in M$

$$
a \equiv b \text { if and only if } W(B, a)=W(B, b) \text { for any } B \in K
$$

$\equiv$ is an equivalence relation and the quotient set $M / \equiv$ is finite, as proved in [2]. We write $[a]$ for the equivalence class which contains $a$ for any $a \in M$. Define a partial ordering $\leqq^{*}$ over $M / \equiv$ by $[a] \leqq^{*}[b]$ if and only if $W(B, a)=t$ implies $W(B, b)=t$ for any $B \in K$.

Of course, $\varliminf^{*}$ is well-defined. Now, we write $N$ for this Kripke model. It can be proved similarly as in [2] that $A \notin L(N)$. We will show $w^{*}(N) \leqq n$. Suppose $w^{*}(N)=m>n$. Since $N$ is finite, $n$ must be also finite. Then $c([a])=m$ for some $[a]$ in $N$. Let $\left[a_{1}\right], \ldots,\left[a_{m}\right]$ be distinct elements in $I([a])$ such that each $\left[a_{\imath}\right]$ is the representative of each equivalence class by $\cong$. We define a subset $H_{i}$ of $N$ by

$$
\begin{aligned}
& H_{i}=\{[c] ;[a] \leqq *[c], \text { and }[d] *[c] \text { for any }[d] \\
&\text { such that } \left.[d] \cong\left[a_{i}\right]\right\} \quad(1 \leqq i \leqq m) .
\end{aligned}
$$

Suppose $[d] \cong\left[a_{i}\right]$ and $[c] \in H_{i}$. Since $[d] \nsubseteq *[c]$, there exists a formula $B_{[c][d]}$ in $K$ such that $W\left(B_{[c][d]}, d\right)=t$ but $W\left(B_{[c][d]}, c\right)=f$. Define $C_{[d]}=\bigwedge_{[c] \in H_{i}} B_{[c][d]}$. Then $C_{[d]}$ is in $K$. Define a formula $D_{i}$ by $D_{i}=\bigvee C_{[d]} C_{[d]}$, where the disjunction is taken for all $[d]$ in $I([a])$ such that $[d] \cong\left[a_{i}\right]$. Remark that each $D_{i}$ may not be in $K$. Then we can prove the following lemma.

Lemma 2.7. Let $b \geqq a$. Then $W\left(D_{i}, b\right)=t$ if and only if $[c] \cong\left[a_{i}\right]$ and $[c] \leqq *[b]$ for some $[c] \in I([a])$.

Using Lemma 2.7, we can show that

$$
W\left(\bigwedge_{i=1}^{m}\left(\neg D_{i} \equiv \bigvee_{j \neq i} D_{j}\right) \supset \bigvee_{i=1}^{m} D_{i}, a\right)=f
$$


(Notice that for each $i \leqq m$, there is a formula $E_{i} \in K$ such that $\neg D_{i} \equiv E_{i}$ is provable in $L J$.) But this contradicts the assumption that each substitution instance of $B_{m-1}$ is valid in $(M, W)$, since $m-1 \geqq n$. Thus $w^{*}(M)$ is not greater than $n$. Now it is clear that $L J+B_{n}>\subset \bigcap_{k<\omega} L\left(M_{k}\right)$.

\section{§3. Disjunction Property}

It is well known that for any formula $A, B$, if $A \vee B \in L J$ then either $A \in L J$ or $B \in L J$. But $L K$ has not this property.

Definition 3.1. A logic $L$ is said to have the disjunction property when for any formula $A, B$, if $A \vee B \in L$ then either $A \in L$ or $B \in L$.

Many of intuitionistic mathematics have this property and it is thought that this is one of the characteristic properties of the intuitionism. So Lukasiewicz [9] conjectured that $L J$ is the only intermediate logic that has the disjunction property. But Kreisel-Putnam [8] answered this negatively. Indeed, they showed that the logic

$$
L J+(\neg p \supset q \vee r) \supset(\neg p \supset q) \vee(\neg p \supset r)
$$

has the disjunction property. Recently, Gabbay-de Jongh [3] proved that there is a strictly descending sequence of intermediate logics $\left\{D_{m}\right\}$ such that each $D_{m}$ has the disjunction property and $\bigcap_{m<\omega} D_{m}>\subset L J$. Anderson [1] investigated also about a class of infinite number of the intermediate logics (in $\mathscr{R}_{\omega \omega}$ ), each of which has the disjunction property. As a corollary of his result, we can show that the class of all the intermediate logics having the disjunction property is not closed under the union (of logics). Clearly, it is not closed under the intersection. At present, we can't seize the general features of the logics having the disjunction property. In this section, we will investigate about certain relations between the classifications of logics and the disjunction property.

Theorem 3.2. $L J+B_{n}$ has the disjunction property if $n \geqq 2$.

Proof. Suppose that neither a formula $A$ nor a formula $B$ is in 
$L J+B_{n}$. By Theorem 2.10 in Ono $[10]$ and Theorm 2.6, there are finite Kripke models $M$ and $M^{\prime}$ of the form $S_{1} \uparrow N$ such that 1) $A \notin L(M)$ and $B \notin L\left(M^{\prime}\right)$ and 2) $w^{*}(M), w^{*}\left(M^{\prime}\right) \leqq n$. Now, define a Kripke model $N$ by $N=S_{1} \uparrow\left(M, M^{\prime}\right)$. Then it is obvious that $N$ is finite and $A \vee B$ $\notin L(N)$. Moreover, we have that $w^{*}(N)=\max \left\{2, w^{*}(M), w^{*}\left(M^{\prime}\right)\right\} \leqq n$, since $n \geqq 2$. Thus $A \vee B \notin L J+B_{n}$ by Theorem 2.6.

In contrast with this result, one can verify that no logics in $\mathscr{T}_{1}$ have the disjunction property, since $L \in \mathscr{T}_{1}$ if and only if $L \ni \neg p \vee \neg \neg p$.

Next, we will show that no logics in finite slices have the disjunction property. We must make some preparations. Hosoi introduced the $\Delta$-projection in Definition 7.1 of $[4]$ and 1.8 of $[5]$. We define here another operation, called the $\nabla$-projection. As we see later, it has the same effect as the $\Delta$-projection. For the definition of the $\nabla$-projection, we get a hint from Smorynski [13].

Definition 3.3. Let $A$ be any formula and $p$ be a propositional variable not appearing in $A$. Then $\nabla_{p}(A)=p \vee(p>A)$.

In the following we regard $p$ as a variable not appearing in $A$, whenever we write $\nabla_{p}(A)$. Sometimes we omit the subscript $p$. Let $p_{1}, \cdots, p_{n}, \ldots$ be a list of propositional variables not appearing in a formula $A$. Define $\nabla^{n}(A)$ by

$$
\begin{aligned}
& \nabla^{0}(A)=A, \quad \text { and } \\
& \nabla^{k+1}(A)=\nabla_{p_{k+1}}\left(\nabla^{k}(A)\right) \quad \text { for } k \geqq 0 .
\end{aligned}
$$

Lemma 3.4. $L J+\nabla^{n}(A)>C L J+\Delta^{n}(A) \quad(n \geqq 0)$ for any formula $A$, where $\Delta^{0}(A)=A$. (See $[4]$.)

Proof. First, we prove that for any formula $B$

1) $\nabla(B) \supset \Delta(B) \in L J \quad$ and

2) $L J+\Delta(B) \ni \nabla(B)$,

where $\nabla(B)=p \vee(p \supset B)$ and $\Delta(B)=((p \supset B) \supset p) \supset p$. It is easy to 
see that $p \supset(((p \supset B) \supset p) \supset p) \in L J$ and $(p \supset B) \supset(((p \supset B) \supset p) \supset p) \in L J$. Thus $\nabla(B) \supset \Delta(B) \in L J$. For 2), $L J+\Delta(B) \ni((\nabla(B) \supset B) \supset \nabla(B)) \supset \nabla(B)$. Since $(\nabla(B) \supset B) \supset \nabla(B) \in L J$, we have $L J+\Delta(B) \ni \Delta(B)$. Now, we prove by induction on $n$ that $L J+\nabla^{n}(A) \ni \Delta^{n}(A)$. It suffices to prove that $\nabla^{n}(A) \supset \Delta^{n}(A) \in L J$. It is trivial that $\nabla^{0}(A) \supset \Delta^{0}(A) \in L J$. Suppose that it holds for $n=k$. Then $\Delta\left(\nabla^{k}(A)\right) \supset \Delta^{k+1}(A) \in L J$. By 1$)$, $\nabla^{k+1}(A) \supset \Delta\left(\nabla^{k}(A)\right) \in L J$. Thus $\nabla^{k+1}(A) \supset \Delta^{k+1}(A) \in L J$. We next show that $L J+\Delta^{n}(A) \ni \nabla^{n}(A)$. It is trivial that $L J+\Delta^{0}(A) \ni \nabla^{0}(A)$. Suppose that it holds for $n=k$. Then there are formulas $B_{1}, \ldots, B_{m}$ such that each $B_{i}$ is a substitution instance of $\Delta^{k}(A)$ and $\bigwedge_{i=1}^{m} B_{i} \supset \nabla^{k}(A) \in L J$. Without loss of generality, we may suppose that the variable $p_{k+1}$ does not appear in any $B_{i}$. Then we have $\bigwedge_{i=1}^{m} \nabla\left(B_{i}\right) \supset \nabla^{k+1}(A) \in L J$. By 2), $L J+\bigwedge_{i=1}^{m} \Delta\left(B_{i}\right)$ $\ni \bigwedge_{i=1}^{m} \nabla\left(B_{i}\right)$ and hence $L J+\bigwedge_{i=1}^{m} \Delta\left(B_{i}\right) \ni \nabla^{k+1}(A)$. As each $\Delta\left(B_{i}\right)$ is a substitution instance of $\Delta^{k+1}(A)$, we have $L J+\Delta^{k+1}(A) \ni \nabla^{k+1}(A)$.

Definition 3.5. For any intermediate logic $L$, we write $\nabla(L)$ for the logic obtained by adding axiom schemata $\nabla(A)$ for each $A \in L$ to the intuitionistic logic LJ. Define $\nabla^{n}(L)$ by $\nabla^{0}(L)=L$ and $\nabla^{k+1}(L)=\nabla\left(\nabla^{k}(L)\right)$ for $k \geqq 0$.

Corollary 3.6. For any intermediate logic $L, \nabla^{n}(L)>\subset \Delta^{n}(L)$ for $n \geqq 0$.

Theorem 3.7. If an intermediate logic is in some finite slice, then it has not the disjunction property.

Proof. We first note that $L J+P_{n}=L J+\Delta^{n}\left(p_{0}\right)>C L J+\nabla^{n}\left(p_{0}\right)$ by Lemma 3.4. Suppose that $L \in \mathscr{S}_{m} \quad(m<\omega)$ and $L$ has the disjunction property. Then $\nabla^{m}\left(p_{0}\right) \in L$ and hence either $p_{m} \in L$ or $p_{m} \supset \nabla^{m-1}\left(p_{0}\right) \in L$. In the former case, we have a contradiction immediately. If the latter is the case then $\nabla^{m-1}\left(p_{0}\right) \in L$. But this contradicts the assumption that $L \in \mathscr{S}_{m}$. Thus $L$ has not the disjunction property.

Next, we extend the above discussion to more general cases. 
Theorem 3.8. An intermediate logic $L$ has not the disjunction property, if there is an intermediate logic $L^{\prime}$ such that $L^{\prime} \not \subset L$ and $\nabla^{n}\left(L^{\prime}\right) \bigcap(L J+\neg p \vee \neg \neg p) \subset L$ for some $n \geqq 1$.

Proof. Suppose that $L$ has the disjunction property. We first prove that $L^{\prime} \bigcap(L J+\neg p \vee \neg \neg p) \not \subset L$. Suppose otherwise. Then for each formula $A$ in $L^{\prime}, A \vee \neg p \vee \neg \neg p \in L^{\prime} \backslash(L J+\neg p \vee \neg \neg p) \subset L$. By the assumption, either $A \in L$ or $\neg p \in L$ or $\neg \neg p \in L$. But in the latter two cases, we have a contradiction. Thus $A \in L$. Hence $L^{\prime} \subset L$. But this contradicts the hypothesis. So, there must exist such $n(\geqq 1)$ that

$$
\begin{aligned}
& \nabla^{n}\left(L^{\prime}\right) \bigcap(L J+\neg p \vee \neg \neg p) \subset L \quad \text { and } \\
& \nabla^{n-1}\left(L^{\prime}\right) \bigcap(L J+\neg p \vee \neg \neg p) \not \subset L .
\end{aligned}
$$

Then for any $A \in L^{\prime}, \quad \nabla^{n}(A) \vee \neg p \vee \neg \neg p \in L$. We have $\nabla^{n}(A) \in L$ similarly as the above proof. Since $p_{n} \notin L, p_{n} \supset \nabla^{n-1}(A) \in L$ and hence $\nabla^{n-1}(A) \in L$. Thus $\nabla^{n-1}\left(L^{\prime}\right) \subset L$. But this leads to a contradiction. So, $L$ has not the disjunction property.

This result can cover many cases which we have already known, as shown in the following.

Corollary 3.9. If an intermediate logic $L$ satisfies one of the following conditions, then $L$ has not the disjunction property.

1) $L \in \mathscr{T}_{1}$.

2) $L$ is not proper in $\mathscr{S}_{\omega}$ (cf. [5]).

3) There is an intermediate logic $L^{\prime}$ such that $L^{\prime} \not \subset L$ and $\nabla^{n}\left(L^{\prime}\right) \subset L$ for some $n \geqq 1$.

We can verify that the $\nabla$-projection is injective and that $\nabla(L) \in \mathscr{R}_{\omega \omega}$ if $L \in \mathscr{R}_{\omega \omega}$. By the above corollary and the remark of the example 3 ) in $\S 1$, we can show that $\mathscr{R}_{\omega \omega}$ contains uncountably many logics which have not the disjunction property. 


\section{References}

[1] Anderson, J. G., Some results in superconstructive propositional calculi, Ph. D. thesis, Univ. of Newcastle upon Tyne, 1966.

[2] Gabbay, D. M., The decidability of the Kreisel-Putnam system, J. Symbolic Logic, 35 (1970), 431-437.

[3] Gabbay D.M. and D.H.J. de Jongh, A sequence of decidable finitely axiomatizable intermediate logics with the disjunction property, to appear.

[4] Hosoi, T., On intermediate logics I, J. Fac. Sci. Univ. Tokyo, Sect. I, 14 (1967), 293-312.

[5] On intermediate logics II, Ibid. 16 (1969), 1-12.

[6] Hosoi T. and H. Ono, The intermediate logics on the second slice, Ibid. Sect. IA, 17 (1970), 457-461.

[7] Jankov, V. A., Constructing a sequence of strongly independent superintuitionistic propositional calculi, Soviet Math. Dokl. 9 (1968), 806-807.

[8] Kreisel G. and H. Putnam, Eine Unableitbarkeitsbeweismethode für den intuitionischen Aussagenkalkül, Arch. Math. Logik Grundlagenforsch. 3 (1957), 74-78.

[9] Lukasiewicz, J., On the intuitionistic theory of deduction, Indag. Math.; Koninklijke Nederlandse Akademie van Wetenschappen Proceedings, Series A, No. 3 (1952), 202-212.

[10] Ono, H., Kripke models and intermediate logics, Publ. RIMS, Kyoto Univ. 6 (1970), 461-476.

[11] - On the finite model property for Kripke models, Ibid. 7 (1971), 85-93.

[12] Segerberg, K., Propositional logics related to Heyting's and Johansson's, Theoria, 34 (1968), 26-61.

[13] Smorynski, C., Intermediate Logics, Unpublished report, 1971. 